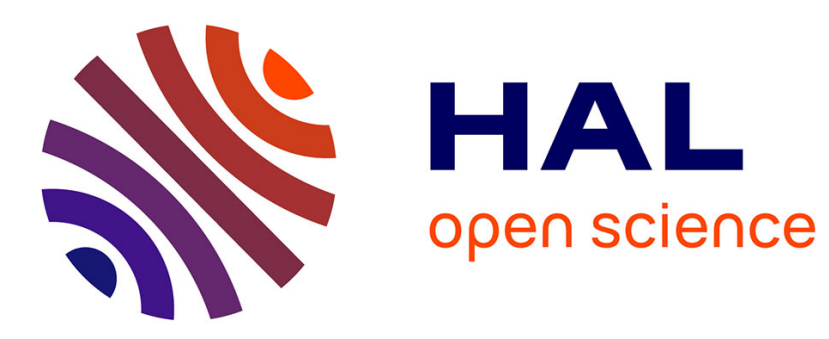

\title{
Analyse des échos ultrasonores par ondelettes
}

S. Moubarik, D. de Vadder, Ph. Benoist

\section{- To cite this version:}

S. Moubarik, D. de Vadder, Ph. Benoist. Analyse des échos ultrasonores par ondelettes. Journal de Physique IV Proceedings, 1994, 04 (C5), pp.C5-1375-C5-1378. 10.1051/jp4:19945306 . jpa-00253021

\section{HAL Id: jpa-00253021 https://hal.science/jpa-00253021}

Submitted on 1 Jan 1994

HAL is a multi-disciplinary open access archive for the deposit and dissemination of scientific research documents, whether they are published or not. The documents may come from teaching and research institutions in France or abroad, or from public or private research centers.
L'archive ouverte pluridisciplinaire HAL, est destinée au dépôt et à la diffusion de documents scientifiques de niveau recherche, publiés ou non, émanant des établissements d'enseignement et de recherche français ou étrangers, des laboratoires publics ou privés. 


\title{
Analyse des échos ultrasonores par ondelettes
}

\author{
S.E. MOUBARIK, D. DE VADDER et Ph. BENOIST* \\ Laboratoire de Mécanique, URA D0850 du CNRS, Ecole Centrale Paris, Grande Voie des Vignes, \\ 92295 Châtenay Malabry, France \\ ${ }^{*}$ CEA/CEREM/STA/LCUS, CEN-Saclay, 91191 Gif-sur-Yvette cedex, France
}

\begin{abstract}
We investigate the time-frequency localization of the continuous wavelet transform as a tool for extracting useful informations about targets from their ultrasonic echoes. A disbond problem is presented as an example. We also present and discuss the use of experimental ultrasonic echoes as analyzing wavelets. This technique is used to study the effect of targets geometry on the received echoes.
\end{abstract}

\section{INTRODUCTION}

Il a déjà été montré que l'analyse par ondelettes peut être utile en contrôle non destructif par échographie $[1,2]$. Nous nous intéressons ici à l'analyse des échos ultrasonores large bande en émission-réception. Ces échos contiennent toute l'information rencontrée sur leur trajet traducteur-milieu-cible-milieu-traducteur. Afin d'extraire celle caractérisant la cible, nous exploitons les propriétés de la transformation en ondelettes continue [3] pour établir des signatures temps-fréquence.

Dans la pratique, les échos sont échantillonnés avec un pas temporel $\Delta$, et la transformation en ondelettes continue est approximée par sa version discrète. Le choix $a=2^{j+m / M}\left(v_{0} / v_{1}\right), 0 \leq m<M, j=0,1, \ldots$, et $b=n \Delta$ présente plusieurs avantages. La stabilité de l'algorithme de reconstruction, équivalente à la condition d'encadrement (ou "frame") des ondelettes [3], est contrôlée simplement par le nombre de voix par octave $M$. Le réseau discret des points $(a, b)$ du demi-plan peut être vu comme une superposition de $M$ réseaux discrets dyadiques, et par conséquent un algorithme rapide inspiré du cas orthogonal peut être utilisé. Par ailleurs, le fait de prendre $b=n \Delta$ (au lieu de $b=a n \Delta$ ) permet de restaurer la propriété d'invariance par translation de la transformée, qui est primordiale si on s'intéresse à la reconnaissance des signatures temps-fréquence [4]. Enfin, l'introduction d'une fréquence $v_{1} \leq(2 \Delta)^{-1}$ rend plus clair, du point de vue de l'utilisateur, le choix de l'intervalle fréquentiel à considérer.

\section{PROBLÈME DE COLLAGE}

L'échantillon étudié est formé de deux plaques d'acier austénitique d'épaisseur $1 \mathrm{~mm}$ chacune, collées ensemble. L'échantillon est insonifié perpendiculairement au plan d'assemblage, et le problème posé est celui de détecter les zones de mauvais collage. Dans la figure 1, nous avons représenté deux échos de cette acquisition avec leurs spectres de Fourier. L'un provient d'une région saine et l'autre d'une zone mal collée. On peut difficilement les distinguer sur cette figure, et cela montre les limitations des représentations uniquement temporelles ou fréquentielles. Nous avons utilisé l'ondelette analysante de Morlet tronquée $g(t)=\pi^{-1 / 4} e^{\mathrm{i} 2 \pi v_{0} t} e^{-t^{2} / 2}$. Cette figure montre le module de la transformée des deux échos dans le même sous-domaine temps-fréquence. On distingue clairement sur cette figure l'existence, dans un 
cas seulement, de contributions cohérentes qui se situent dans le temps entre les réflexions multiples du signal émis. L'emplacement de ces contributions à la même fréquence que l'écho principal et leur décalage constant dans le temps confirme qu'elles proviennent de l'interface décollée.
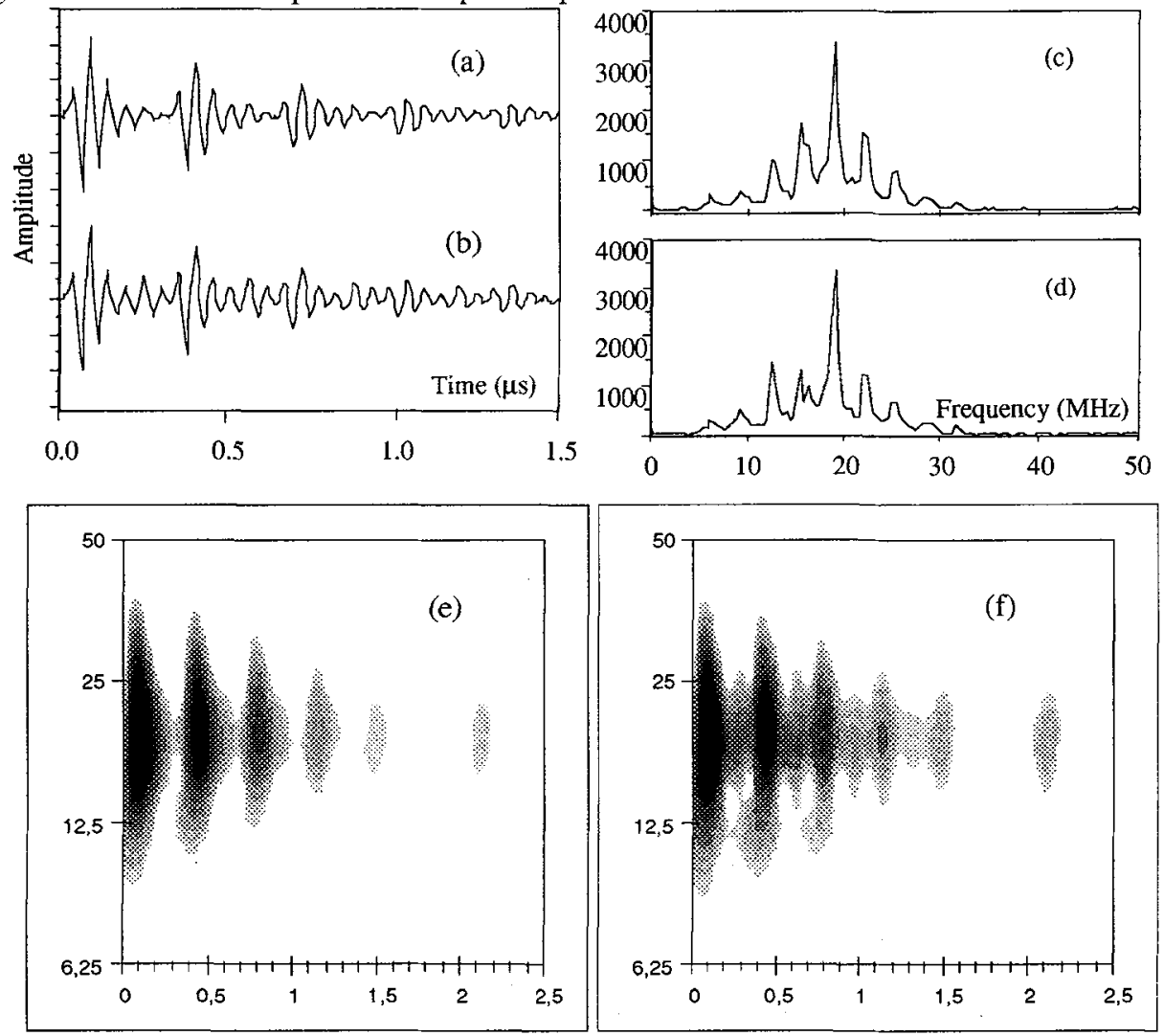

Fig. 1 - Échos réfléchis dans une pièce formée de deux plaques d'acier austénitique $(1 \mathrm{~mm}$ d'épaisseur chacune) collées ensemble. (a) écho provenant d'une zone bien collée. (b) écho provenant d'une zone mal collée. (c) et (d) leurs spectres de Fourier respectifs. (e) et (f) le module de leur transformée en ondelettes. Chaque niveau de gris représente une dimimution de $2 \mathrm{~dB}$ par rapport au maximum absolu.

\section{ONDELETTES EXPÉRIMENTALES}

La seconde application que nous présentons entre dans le cadre de l'identification des géométries à l'origine des échos. Plusieurs études ont déjà été menées dans ce cadre afin de comprendre le mécanisme de formation des échos et la différence d'information qui existe lorsqu'ils sont réfléchis par des cibles de géométrie différente [5]. Nous avons voulu savoir s'il était possible de caractériser des cibles de géométrie simple par leur signature temps-fréquence. Mais comme nous l'avons mentionné précédemment, le choix de l'ondelette optimale pour l'analyse d'un signal donné est un problème encore ouvert. On sait que l'écho tel qu'observé sur un oscilloscope est un double produit de convolution de l'information électro-acoustique du traducteur avec celle géométrique du milieu de propagation et celle de la cible. Nous avons pensé alors que si l'ondelette analysante avait une connaissance a priori du traducteur et/ou du milieu de propagation elle pourrait donner une signature plus caractéristique de la cible. Nous avons donc utilisé comme ondelette analysante des signaux expérimentaux de référence et nous avons comparé les résultats avec ceux obtenus par l'ondelette de Morlet. La première difficulté à utiliser un signal expérimental vient de la condition d'admissibilité [3]. Cependant, pour un signal suffisamment régulier, cette condition est équivalente à exiger qu'il soit de moyenne nulle. D'un point de vue physique ceci doit être toujours le cas pour les échos ultrasonores. On a donc juste à corriger les effets de la quantification lors de l'acquisition. Un 
signal analysant
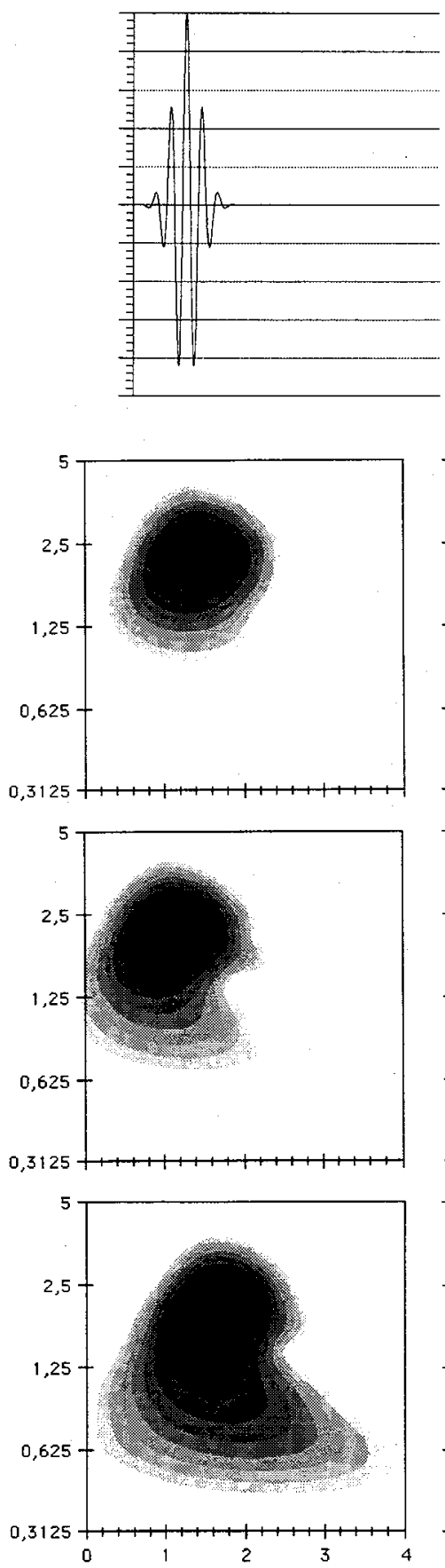

Écho sur disque $\varnothing 2 \mathrm{~mm}$
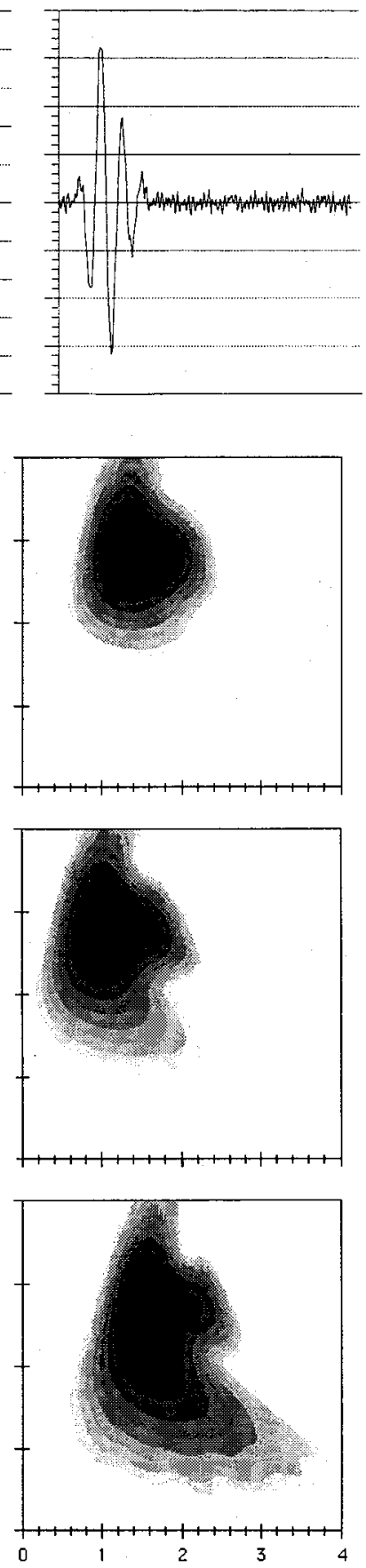

Signal de référence
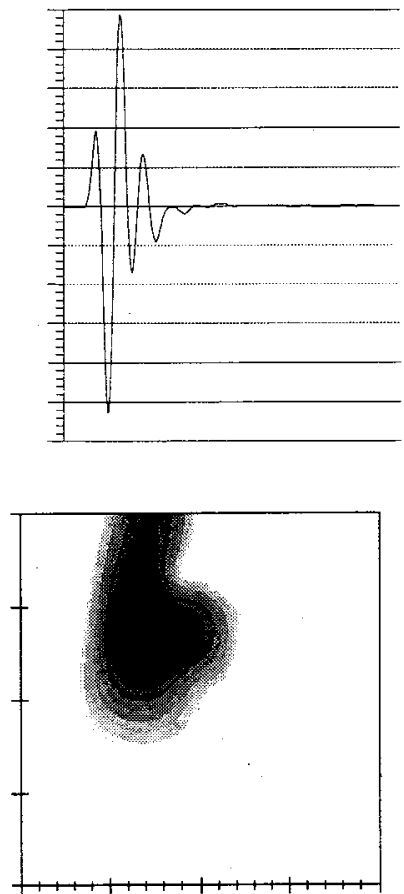

Disque

$\varnothing 5 \mathrm{~mm}$

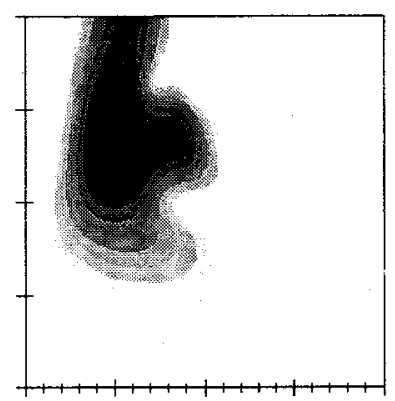

Bille

$\varnothing 8 \mathrm{~mm}$

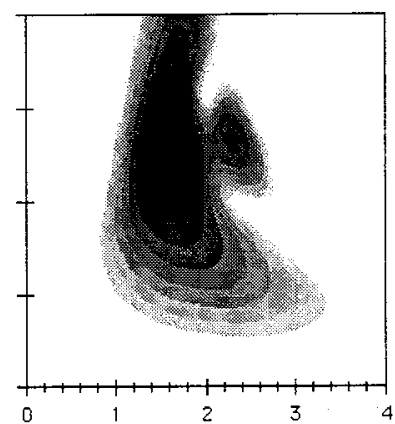

Cible

$\mathrm{mm}$
Cône

Fig. 2 - Traducteur large bande $\varnothing 25,4 \mathrm{~mm}$, fréquence centrale $2,25 \mathrm{MHz}$, distance $400 \mathrm{~mm}$.

Module de la transformée en ondelettes des échos reçus d'un disque $\emptyset 5 \mathrm{~mm}$, d'une bille $\emptyset 8 \mathrm{~mm}$ et d'un cône semi-infini d'ouverture $160^{\circ}$. Le signal analysant est représenté en première ligne. Les échelles temporelles (en $\mu \mathrm{s}$ ) et fréquentielles (en $\mathrm{MHz}$ ) sont identique pour toute la figure. 
autre point important est celui de l'analyticité de l'ondelette analysante. En effet, pour bien illustrer ce point, considérons le signal $f(t)=\cos (\omega t)$. Sa transformée en ondelettes s'écrit $T_{f}(a, b)=\sqrt{a} / 2\left(e^{i \omega b} \overrightarrow{\hat{g}}(a \omega)+e^{-i \omega b} \overline{\hat{g}}(-a \omega)\right)$. Si l'ondelette analysante $g$ est analytique, c'est-à-dire $\hat{g}(\omega)=0$ pour $\omega<0$, la fréquence du signal est directement donnée par la phase de la transformée, et le module $\left|T_{f}(a, b)\right|$ reste constant le long des lignes $a=$ cte. Ceci n'est évidemment plus vrai si $g$ n'est pas analytique puisqu'il y aura interférence entre les deux termes de la somme. Pour avoir une ondelette expérimentale analytique il suffit de considérer le signal $g(t)=s(t)+\mathrm{i} H[s](t)$, où $s(t)$ est le signal expérimental et $H[s](t)$ sa transformée de Hilbert. En effet, une des propriétés des fonctions analytiques est que leur partie réelle et leur partie imaginaire sont transformées de Hilbert l'une de l'autre. L'ondelette ainsi obtenue n'est en général pas centrée autour de l'origine, nous les avons recentré systématiquement. Sur la figure 2 nous avons représenté le module de la transformée en ondelettes de trois cibles de géométrie simple : a) un disque de $5 \mathrm{~mm}$ de diamètre, b) une bille de $8 \mathrm{~mm}$ de diamètre et, c) un cône semi-infini de $160^{\circ}$ d'angle d'ouverture. Le traducteur large bande utilisé est un disque de $25,4 \mathrm{~mm}$ de diamètre et de fréquence centrale 2,25 MHz. Les cibles sont placées normalement au traducteur à une distance de 400 mm sur l'axe. Les ondelettes analysantes utilisées sont respectivement : 1) l'ondelette de Morlet tronquée, 2) l'écho réfléchi sur un petit disque de $2 \mathrm{~mm}$ de diamètre dans exactement les mêmes conditions que les cibles étudiées et, 3) l'écho réfléchi sur un plan quasi-infini, placé très près du traducteur (à $8 \mathrm{~mm}$, centré et parallèle au traducteur), que l'on considère comme le signal tenant compte des réponses électro-acoustique et acousto-électrique du traducteur (que nous appelons signal de référence) [6]. On constate que la signature temps-fréquence d'une cible donnée n'est qualitativement pas très influencée par l'ondelette analysante choisie. Nous pensons que la différence constatée par exemple dans le cas du cône est due plus à la différence de la localisation temps-fréquence des ondelettes utilisées qu'à leur contenu physique. Mais ceci reste à confirmer par une étude plus systématique. Remarquons aussi que la localisation tempsfréquence optimale, obtenue pour l'ondelette de Morlet, n'est pas toujours la plus adaptée. Quant à la différence entre la signature des différentes cibles, là aussi on retrouve qualitativement les mêmes différences quelle que soit l'ondelette analysante. On constate que les composantes basse fréquence augmentent en allant du disque au cône, en passant par la sphère. On remarque aussi que ces composantes basse fréquence suivent un profil assez particulier. Dans le cas du cône, on remarque l'apparition d'une seconde contribution au niveau du pic central, décalée dans le temps et un peu plus basse fréquence. Cette contribution est moins bien séparée dans le cas des autres cibles.

\section{CONCLUSION}

Nous avons présenté deux applications de l'analyse par ondelettes. Nous avons trouvé cet outil bien adapté aux problèmes du contrôle non destructif par ultrasons. Les signatures temps-fréquence obtenues par cette méthode sont riches mais leur interprétation est parfois délicate.

\section{REMERCIEMENTS}

Nous remercions A. Lhémery pour les nombreuses discussions que nous avons eues lors de cette étude.

\section{RÉFÉRENCES}

[1] Lefebvre J. P., Lasaygues P., “Application de l'analyse en ondelettes à la détection de fissures par échographie ultrasonore", 2ème Congrès Français d'Acoustique, Vol. II, J. Roux Ed., Arcachon France avril 1992 (Les Éditions de Physique, Les Ulis, 1992) pp. 637-640.

[2] Moubarik S.E., De Vadder D., Benoist Ph., "Wavelets and nondestructive evaluation", Review Of Progress in QNDE, Vol. 12A, D.O. Thompson and D.E. Chimenti Eds. La Jolla CA. July 1992 (Plenum Press, New York, 1993), pp. 727-734.

[3] Daubechies I., Ten Lectures on Wavelets (CBMS-NSF Series in Appl. Math., SIAM, 1992), et les références incluses.

[4] Mallat S., and Zhong S., Characterization of Signals from Multiscale Edges, Technical Report No. 592, New York University, 1991.

[5] Raillon R., Lhémery A. De Vadder D., "Extension du modèle de Freedman de formation des échos au cas transitoire", Ces proceedings.

[6] Lhémery A., and Raillon R., à paraitre dans J. Acoust. Soc. Am., 1994. 